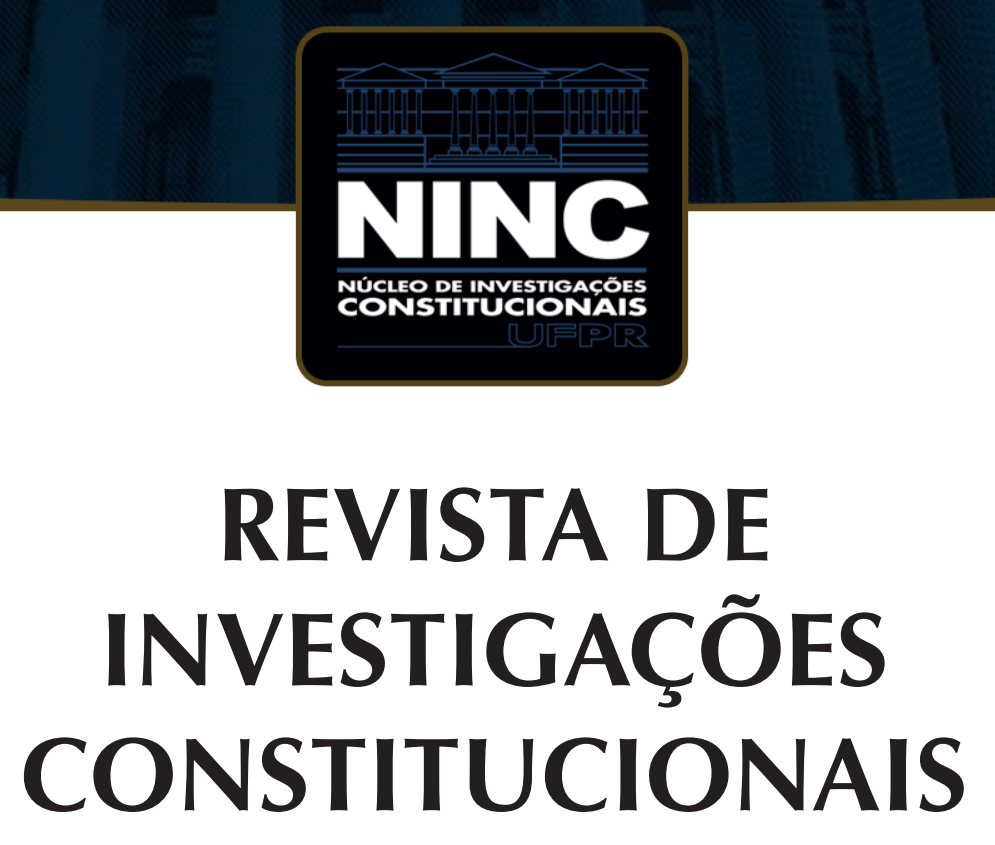

JOURNAL OF CONSTITUTIONAL RESEARCH

vol. 7 | n. 1 | janeiro/abril 2020 | ISSN 2359-5639 | Periodicidade quadrimestral Curitiba | Núcleo de Investigações Constitucionais da UFPR | www.ninc.com.br 


\title{
El constitucionalismo del ius commune a través de los derechos humanos: los avances y resistencias en el ámbito mexicano
}

\section{The constitutionalism of the ius commune through human rights: the advances and resistances in the Mexican sphere}

\author{
JOSÉ DE JESÚS BECERRA RAMÍREZ ${ }^{\text {l, * }}$ \\ 'Universidad de Guadalajara (Guadalajara, Jalisco, México) \\ jbecerra33@hotmail.com \\ https://orcid.org/0000-0002-6419-185X \\ Recebido/Received: 11.02 .2019 / February $11^{\text {th }}, 2019$ \\ Aprovado/Approved: $13.07 .2020 /$ July $13^{\text {th }}, 2019$
}

Resumen

El presente ejercicio académico se proyecta con el fin de realizar una aproximación modesta, hacia el camino que ha emprendido el constitucionalismo contemporáneo en la búsqueda de un ius commune, que si bien es cierto se pudiera cimentar en tres aspectos fundamentales, a saber: los derechos humanos, al Estado de derecho y a la democracia; sin embargo, en este análisis se enfoca principalmente al primero de ellos, además de analizar cómo se ha planteado en el ordenamiento jurídico mexicano, sobre todo lo relacionado con sus avances normativos y las resistencias en la práctica cotidiana.

Palabras clave: ius commune; derechos humanos; constitucionalismo; Estado de Derecho; México.
Abstract

This academic work is projected in order to make a modest approach, to the path that contemporary constitutionalism has undertaken in the search for a ius commune, which, although it is true, could be based on three fundamental aspects: the human rights, the rule of law, and democracy; However, this analysis focuses mainly on the first of them, in addition to analyzing how it has been proposed in the Mexican legal system, especially regarding its normative advances, and resistance in daily practice.

Keywords: Ius commune; human rights; constitutionalism; Rule of Law; Mexico.

Como citar esse artigo/How to cite this article: BECERRA RAMÍREZ, José de Jesús. El constitucionalismo del ius commune a través de los derechos humanos: los avances y resistencias en el ámbito mexicano. Revista de Investigações Constitucionais, Curitiba, vol. 7, n. 1, p. 87-105, jan./abr. 2020. DOl: 10.5380/rinc.v7i1.64878.

" Profesor investigador titular C en la Universidad de Guadalajara (Guadalajara, Jalisco, México). Doctor en Estudios Avanzados en Derechos Humanos por la Universidad Carlos III de Madrid. Miembro del Sistema Nacional de Investigadores reconocido por el Consejo Nacional de Ciencia y Tecnología de México. Actualmente Director del Instituto de Investigación y Capacitación en Derechos Humanos adscrito a la Comisión Estatal de Derechos Humanos Jalisco, México. E-mail: jbecerra33@hotmail.com. 


\section{SUMARIO}

1. Introducción; 2. El camino para un ius commune; 3. Referencia al caso mexicano; 4. Avances y resistencias; $\mathbf{5}$. Conclusiones; $\mathbf{6}$. Referencias.

\section{INTRODUCCIÓN}

El constitucionalismo que empezó a gestarse después de lo acontecido en la Segunda Guerra Mundial, el cual dio un giro significativo en su configuración y que se extendió de igual manera al Derecho internacional, en razón de que por un lado, se caracterizó por la superación del monopolio de la producción jurídica, al no delimitarse a las fronteras de los Estados nacionales, y por otro parte, aparecen diversos sujetos del Derecho internacional, como lo son organizaciones colectivas internacionales e incluso de forma incipiente los propios individuos. Todo lo cual, llevó al inicio de una gestación de cierta unificación jurídica en algunas áreas, especialmente en el campo de los derechos humanos. ${ }^{1}$

Por lo anterior, se habla de una evolución y transformación, como bien lo apunta Armin Von Bogdandy, al señalar de cómo ha cambiado el ámbito del poder público, al resaltar que esté se delimitaba originalmente al Estado, pero que actualmente ha cambiado, por motivo que las instituciones supranacionales e internacionales cada día están incidiendo de forma por demás relevante en las relaciones de índole social y en el campo del poder político. Tal circunstancia lleva a replantear la idea tradicional de la soberanía nacional, en el cual los Estados se jactaban de ser entes independientes, de ahí, que sin perder esta característica, la nueva realidad contemporánea si la modifica, pues las estructura del poder tradicional del Estado, sobre todo en lo que a derechos humanos se refiere, si la socava, pues tanto su reconocimiento y sus medios de garantía, no son de fuente exclusiva de los ámbitos nacionales. ${ }^{2}$

Adicionalmente, también es relevante mencionar, que ante la realidad imperante desde la segunda mitad del siglo XX e incluso presente hasta nuestros días, en el que los propios Estados nacionales, se ven impedidos por sí solos resolver plenamente sus problemas locales, debido a la insuficiencia de sus capacidades, por lo que se requiere el concurso de la comunidad internacional. Por ello, se platea un Estado de corte cooperativo y de tendencia abierto con lo internacional, sobre todo en materia de derechos humanos. ${ }^{3}$

RUIZ MIGUEL, Alfonso. Entre Estado y cosmópolis. Madrid:Trotta, 2014. p. 28 y 29.

2 VON BOGDANDY, Armin. lus constitucionale commune latinoamericano. Una aclaración conceptual. In: VON BOGDANDY, Armin; FIX-FIERRO, Héctor; MORALES ANTONIAZZI, Mariela. Ius constitucionale commune en América Latina: rasgos, potencialidades y desafíos. México: UNAM, Max-Plank-Institut Für Ausländishes e Instituto Iberoamericano de Derecho Constitucional, 2014. p. 5.

3 VON BOGDANDY, Armin. lus constitucionale commune latinoamericano. Una aclaración conceptual. In: VON BOGDANDY, Armin; FIX-FIERRO, Héctor; MORALES ANTONIAZZI, Mariela. Ius constitucionale commune 
Así, uno de los dilemas de las nuevas construcciones constitucionales es la de aspirar a situar al constitucionalismo en el camino de una dimensión de índole supranacional, superando aquella idea tradicional del Estado soberano consolidado como Estado de derecho, que por sí mismo pretendía hacer una realidad los derechos humanos sin necesidad de buscar su fundamento en otras latitudes. ${ }^{4}$ Pero que la realidad actual está concibiendo una nueva forma de comprensión del mundo jurídico, en el que muchos ámbitos, según Gustavo Zagrebelsky, se van emancipando llamativamente del territorio, en el que si bien es cierto, existen ciertos principios reconocidos en determinadas constituciones pero con un alcance universal, como es el caso de la dignidad humana y en general los derechos humanos. ${ }^{5}$ Convirtiéndose necesario e imprescindible un orden jurídico internacional bajo la idea de un Estado de derecho internacional que cuente con normas claramente identificables para los sujetos obligados, con órganos de garantías plenamente legitimados que respondan a las exigencias de respeto efectivo para los derechos. ${ }^{6}$ Y que a la vez esté abierto al diálogo entre las diversas culturas, que lleve a un consenso internacional del que surja un marco jurídico que tenga como premisa mayor el respeto y garantía de los derechos. ${ }^{7}$

Por lo tanto, si bien es cierto se ha plateado que el ius constitucionale commune se construye a través de tres pilares específicos, a saber: "el respeto a los derechos humanos, al Estado de derecho y a la democracia". "En el presente ejercicio se realizará un análisis modesto y delimitado solamente a los aspectos relacionados con los derechos humanos, para ver cómo ha sido dicho proceso de asimilación en el ámbito mexicano, sobre todo en su relación con el sistema interamericano.

\section{EL CAMINO PARA UN IUS COMMUNE}

Para el espacio geográfico que ocupa el continente americano en el cual se encuentra incluido México, el que exista un sistema regional de protección de los derechos humanos, es una enorme oportunidad de construcción de un orden supranacional

en América Latina: rasgos, potencialidades y desafíos. México: UNAM, Max-Plank-Institut Für Ausländishes e Instituto Iberoamericano de Derecho Constitucional, 2014. p. 9.

4 FIORAVANTI, Mauricio. Constitucionalismo, experiencia histórica y tendencias actuales. Madrid:Trotta, 2014. p. 142-148.

5 ZAGREBELSKY, Gustavo. La ley y su justicia. Madrid: Trotta, 2014. p. 329-330.

6 DE ASIS, Rafael. Cuestiones de derecho. Bogotá: Universidad del Externado, 2005. p. 80.

7 CORONA NAKAMURA, Luis Antonio; GÚZMAN ROBLEDO, María Teresa. Evolución y desarrollo de los derechos económicos, sociales y culturales. In: MARTíNEZ LAZCANO, Alfonso Jaime. Derechos económicos, sociales y culturales. Bogotá: Ediciones Nueva Jurídica, 2017. p. 63.

8 VON BOGDANDY, Armin. Ius constitucionale commune latinoamericano. Una aclaración conceptual. In: VON BOGDANDY, Armin. FIX-FIERRO, Héctor; MORALES ANTONIAZZI, Mariela. Ius constitucionale commune en América Latina: rasgos, potencialidades y desafíos. México: UNAM, Max-Plank-Institut Für Ausländishes e Instituto Iberoamericano de Derecho Constitucional, 2014. p. 8. 
basado en el respeto y garantía de los derechos de todas las personas. Es decir que, con el establecimiento de la Convención Americana sobre Derechos Humanos y la constitución de la Corte Interamericana, se constituye un marco de enorme relevancia para la instauración de un circulo virtuoso de dialogo entre los entes nacionales e internacionales, que establezcan contenidos básicos y elementales para el reconocimiento de un derecho común interamericano. ${ }^{9}$

Sin embargo, la premisa lanzada en el párrafo anterior no es una tarea fácil, ni sencilla, por lo cual en el presente documento se pretende hacer una aproximación a un camino que iniciado desde 1969, cuando en San José de Costas Rica se adoptó la citada Convención Americana, con categoría de tratado internacional, que incluso previamente fue impulsada por la Declaración Americana de los Derechos y Deberes del Hombre de 1948 por la Organización de Estados Americanos, en la cual se parte de la proclamación de los derechos de la personas, no por ser parte de un determinado país, sino de la condición propia de su naturaleza humana, a los cuales el poder político tiene el fin promover y respetar, tal y como lo expresa en los primeros párrafos de su considerando, al expresar:

Que los pueblos americanos han dignificado la persona humana y que sus constituciones nacionales reconocen que las instituciones jurídicas y políticas, rectoras de la vida en sociedad, tienen como fin principal la protección de los derechos esenciales del hombre y la creación de circunstancias que le permitan progresar espiritual y materialmente y alcanzar la felicidad;

Que, en repetidas ocasiones, los Estados americanos han reconocido que los derechos esenciales del hombre no nacen del hecho de ser nacional de determinado Estado sino que tienen como fundamento los atributos de la persona humana;

Que la protección internacional de los derechos del hombre debe ser guía principalísima del derecho americano en evolución;

Con esta redacción se intentó proyectar de alguna manera el inicio de un derecho común interamericano, basado en los derechos humanos, como un eje esencial que identifica a los diversos países del continente. Pero, que tal intentó declarativo se vino a catalizar con el establecimiento de la Corte Interamericana, pues, bien lo ha expresado el exjuez de la citada Corte, Sergio García Ramírez sobre la relevancia de un órgano de tal naturaleza que realiza un papel es de tal calado, al estar "Ilamado a interpretar y aplicar normas que se proyectan sobre numerosos países y centenares de millones

9 SUÁREZ OSMA, Ingrid. Control de convencionalidad y autoprecedente interamericano. Bogotá: Universidad de la Sabana-Grupo Editorial Ibañez, 2015. p. 16. 
de personas, deben de tomarse conciencia del papel que le incumbe...." Esta idea de García Ramírez, es parte fundamental para comprender el rol que está desempeñando dicho órgano interamericano para la construcción de un especio supranacional guiado por el sendero de los derechos humanos. ${ }^{10}$

Lo expresado, no es otra cosa que el replanteamiento de una nueva cosa pública internacional, que apunta a compartir una dimensión más allá de las fronteras nacionales, basado en la dignidad, la igualdad, la justicia y la paz, tal como lo ha señalado Gustavo Zagrebelsky, al resaltar esta res publica universal que tiene una pertenencia a un pueblo sin confines. Por lo cual las personas independiente del lugar donde se encuentren, reaccionen ante cualquier agravio a ese contenido compartido, por ser los derechos humanos una serie de principios que le son comunes para todos. ${ }^{11}$

Sin embargo, es pertinente resaltar, que dicha idea de un ámbito común no es nueva, en razón de que en el momento actual las recientes construcciones jurídicas lo han retomado. ${ }^{12}$ El ius commune como tal surgió desde los siglos XIII hasta finales del siglo XVIII en Europa, presentándose con la intención de superar al Estado nacional clásico y su monopolio estatista de las fuentes del derecho. Esta circunstancia se debió a dos factores fundamentales: el primero, como consecuencia de la enseñanza impartida siempre y solo en latín, por ser la lengua culta común en el que se escribían los libros de Derecho en toda Europa hasta el siglo XVII y no la lengua de cada país, convirtiendo al latín en el único vínculo común para estudiar Derecho, (de ahí que el contenido bibliográfico utilizado por las universidades europeas fuera el mismo). El segundo factor de gran relevancia fue que el único Derecho que se impartía en las universidades de dicha época era el derecho romano-canónico, por lo que los derechos de cada país no fueron objeto de estudio sino hasta bien entrada la Edad Moderna en los centros educativos, siendo en consecuencia el Corpus iuris civiles y el Corpus iuris canonici los únicos leídos. Por consiguiente, la recepción del Derecho romano-canónico significó en todos los casos la pugna entre un Derecho nuevo y el Derecho viejo o tradicional de cada país. ${ }^{13}$

La tendencia a un Derecho común indica la inclinación a unas ideas jurídicas de similares orientaciones con efectos más allá de las fronteras nacionales clásicas que destaquen en lo fundamental, así como también en aspectos que tienen que ver con

10 GARCÍA RAMÍREZ, Sergio. La Corte Interamericana de Derechos Humanos. México: Porrúa, 2007. p. X.

11 ZAGREBELSKY, Gustavo. La virtud de la duda, una conversación sobre ética y derecho con Geminello Preterossi. Madrid:Trotta, 2012. p. 116-117

12 MORÁN MARTíN, Remedios. El ius commune como antecedente jurídico de la Unión Europea. Cuadernos de Historia del Derecho, n. 12, 2005. p. 99-123.

13 TOMÁS Y VALIENTE, Francisco. Manual de historia de derecho español. Madrid: Tecnos. 2003. p. 198-200. También en: GARCÍA Y GARCÍA, Antonio. Perspectivas del derecho común romano-canónico al filo del año 2000. In: El derecho común y Europa. Jornadas internacionales de historia del derecho del Escorial de 1999. Madrid: Dykinson, 2000. p. 9-20. 
una cultura jurídica y de la cultura en lo general. ${ }^{14}$ Esta idea de concebir un ius commune vuelve a resurgir en la actualidad, como ya se ha apuntado, debido a la consecuencia de las nuevas relaciones internacionales y las concepciones que le acompañan, para formar espacios comunes tanto a nivel regional como universal, que a la vez repercute en las diversas áreas del Derecho. ${ }^{15}$

Así, es precisamente una de las funciones de la jurisprudencia actual el elaborar mediante su ejercicio ese patrimonio que nos es común a toda la humanidad, lo cual se puede encontrar en muchas de las constituciones en el mundo, pero que, principalmente las vamos a identificar en resoluciones, tanto de tribunales nacionales como supranacionales, como puede ser: "la igualdad y la no discriminación de los seres humanos, su dignidad y la prohibición de tratos vejatorios, la protección de las minorías, los derechos de libertad clásicos y los derechos sociales, la democracia y el derecho al autogobierno de las comunidades locales, la protección de las identidades culturales etc.,"16 En consecuencia, la labor de interpretación de dichos principios, puede ser una forma de encontrar las respuestas que nos son comunes a todos.

Es pertinente hacer un ejercicio que clarifique el camino para lograr la idea aquí planteada, de lograr un derecho común en materia de derechos humanos. La encomienda aquí planteada, es algo que puede ser factible y alcanzable en cierta medida, debido a que en los últimos años, a pesar de ciertas resistencias, se han expresado tendencias de diversa índole hacia tal camino, como lo son las adecuaciones normativas realizadas por los Estados nacionales, tal es el caso del ámbito latinoamericano, por sendas reformas constitucionales, con habilitaciones para incorporar el canon del ius commune internacional, mediante cláusulas de apertura hacia ello, al igual que acciones jurisprudenciales, políticas públicas y los claros debates académicos. Tal tendencia la ha llamado Sergio García Ramírez, como una "navegación americana", en el que mediante los derechos humanos se camina hacia un derecho común. Adicionalmente resalta el mencionado jurista, que se incrementa los contenidos para tal fin, por los diversos instrumentos interamericanos que han sido ratificados por Estados de la región mediante protocolos que se suman a la Convención Americana sobre Derechos Humanos, que amplían su alcance. ${ }^{17}$

\footnotetext{
14 HABERLE, Peter; KOTZUR, Markus. De la soberanía al derecho constitucional común: palabras clave para un diálogo europeo-latinoamericana. México: UNAM, 2003. p. 18-19.

15 HABERLE, Peter; KOTZUR, Markus. De la soberanía al derecho constitucional común: palabras clave para un diálogo europeo-latinoamericana. México: UNAM, 2003. p. 18-19. De igual manera en: ALONSO GARCÍA, Ricardo. Derecho comunitario, derechos nacionales y derecho común europeo. Madrid: Civitas, 1989. p. 227.

16 Tal afirmación la realiza Gustavo Zagrebelsky, al señalar de la presencia de un derecho constitucional común, más allá del Estado. ZAGREBELSKY, Gustavo. La ley y su justicia, tres capítulos de justicia constitucional. Madrid: Trotta, 2014. p. 335

17 GARCÍA RAMÍREZ, Sergio. La "navegación americana" de los derechos humanos: hacia un ius commune. In: VON BOGDANDY, Armin; FIX-FIERRO, Héctor; MORALES ANTONIAZZI, Mariela. Ius constitucionale commune
} 


\section{REFERENCIA AL CASO MEXICANO}

En el escenario aquí descrito, y enfocando el análisis hacia México, sin lugar a dudas obliga a analizar cómo ha sido el proceso de apertura constitucional para la incorporación del Derecho internacional al ámbito interno y en consecuencia al ius commune, especialmente lo que se refiere a los catálogos de derechos humanos contenidos en instrumentos internacionales, al igual que la recepción de la jurisprudencia derivada de los tribunales destinados a la garantía de tales derechos. Todo ello, con el fin de fortalecer los derechos reconocidos en la Constitución nacional, los cuales se verían beneficiados con un ejercicio de enriquecimiento mutuo por el manejo del canon internacional de los derechos fundamentales de las personas.

La configuración constitucional en México, se delimitó históricamente hasta fechas recientes en el tema que nos ocupa, en la redacción del artículo 133 de la Carta Magna, el cual tiene su origen en la Constitución de 1917, que a la letra dice: ${ }^{18}$

Esta constitución, las leyes del Congreso de la Unión que emanen de ella y todos los tratados que estén de acuerdo con la misma, celebrados y que se celebren por el Presidente de la República, con aprobación del Senado, serán la Ley Suprema de toda la Unión. Los jueces de cada estado se arreglarán a dicha constitución, leyes y tratados, a pesar de las disposiciones en contrario que pueda haber en las Constituciones o leyes de los estados.

La interpretación dada por el máximo tribunal del país en relación a tal artículo, no fue muy propensa a darle un valor de rango jerárquico constitucional a los tratados internacionales de derechos humanos, sino más bien, lo mantuvo en un grado inferior a la Carta Magna, pues partió originalmente de otorgarle un nivel legal, al equiparar a las leyes federales con los tratados internacionales; circunstancia que se desprende de la tesis aislada del amparo en revisión 2069/91 dictada por el pleno de la Suprema Corte de Justicia de la Nación el 30 de junio de 1992. Posteriormente, en el año de 1999 se vuelve a discutir el tema de la jerarquía de los tratados, en la que se modifica la tesis anterior por el pleno de la Suprema Corte, para ubicarlos por encima de las leyes federales, pero, en un segundo plano respecto a la Constitución. ${ }^{19}$ Sin embargo, el debate no

en América Latina: rasgos, potencialidades y desafíos. México: UNAM, Max-Plank-Institut Für Ausländishes e Instituto Iberoamericano de Derecho Constitucional, 2014. p. 459-467.

18 BECERRA RAMÍREZ, Manuel. Recepción del derecho internacional en el derecho interno. México: Universidad Nacional Autónoma de México, 2006. p. 110.

19 MÉXICO. Suprema Corte de Justicia de la Nación. Tesis P. LXXVII/99. Tratados Internacionales. Se ubican jerárquicamente por encima de las leyes federales y en un segundo plano respecto de la Constitución Federal. Semanario Judicial de la Federación y su Gaceta, Tomo X, nov. 1999, p. 46. Registro 192867, Tesis Aislada, Novena Época, Pleno, Constitucional. Nota: Esta tesis abandona el criterio sustentado en la tesis P. C/92, publicada en la Gaceta del Semanario Judicial de la Federación Número 60, Octava Época, diciembre de 1992. p. 27. 
quedó agotado, toda vez que en el 2007, el mismo órgano de justicia en México, analizó nuevamente el referido aspecto y resolvió en votación dividida el siguiente criterio:

TRATADOS INTERNACIONALES. SON PARTE INTEGRANTE DE LA LEY SUPREMA DE LA UNIÓN Y SE UBICAN JERÁRQUICAMENTE POR ENCIMA DE LAS LEYES GENERALES, FEDERALES Y LOCALES. INTERPRETACIÓN DEL ARTÍCULO 133 CONSTITUCIONAL [...]. ${ }^{20}$

En éste nuevo criterio, el debate se centró más en la incorporación de una nueva categoría de leyes no contenidas en las tesis anteriores, toda vez que se incluyó a las leyes generales dentro del esquema de la jerarquía normativa del ordenamiento nacional. En consecuencia, se dejó de lado un debate amplio respecto a la distinción clara y prevalente de los tratados de derechos humanos en relación con los instrumentos de índole general, es decir, separar por un lado los tratados de derechos humanos para otorgarles un valor equiparable al de la Constitución, y por otro, a los tratados del Derecho internacional general. Más por el hecho que a nivel internacional, muchos de los debates constitucionales más intensos versan sobre la articulación coherente entre dicho ámbito y el nacional en materia de derechos fundamentales. Basta ver, por ejemplo, algunas constituciones que lo han incluido en sus disposiciones, incluso, se ha dado el caso, que se les otorgue a los tratados de derechos humanos un rango supraconstitucional, como es el caso de la Constitución de Guatemala de 1985 y reformada en 1993, que en su artículo 46 menciona:

Preeminencia del Derecho Internacional. Se establece el principio general de que en materia de derechos humanos, los tratados y convenciones aceptados y ratificados por Guatemala tienen preeminencia sobre el derecho interno.

O también la Constitución Colombiana de 1991, que establece:

Los tratados y convenios internacionales ratificados por el Congreso, que reconocen los derechos humanos y que prohíben sus limitaciones en los estados de excepción prevalecen en el orden interno.

Los derechos y deberes consagrados en esta Carta, se interpretarán de conformidad con los tratados internacionales sobre derechos humanos ratificados por Colombia.

Incluso, previamente a las anteriores, en la Constitución de Portugal de 1976, que sirvió de inspiración a la española de 1978 sobre la apertura constitucional a los

20 MÉXICO. Suprema Corte de Justicia de la Nación. Tesis P. IX/2007. Tratados Internacionales. Son parte integrante de la ley suprema de la Unión y se ubican jerárquicamente por encima de las leyes generales, federales y locales. Interpretación del artículo 133 constitucional. Registro n. 172650, Semanario Judicial de la Federación y su Gaceta, Tomo XXV, abr. 2007, p. 6. Tesis Aislada, Novena Época, Pleno, Constitucional. 
instrumentos internacionales de derechos humanos, se señala en el artículo 16 lo siguiente: ${ }^{21}$

1. Los derechos fundamentales consagrados en la Constitución no excluyen cualesquiera otros que consten en las leyes y en las normas aplicables de Derecho internacional.

2. Los preceptos constitucionales y legales relativos a los derechos fundamentales deben de ser interpretados e integrados en armonía con la Declaración Universal de los Derechos Humanos.

Así, las constituciones citadas son claras referencias de los nuevos modelos constitucionales bajo un prototipo de Estado constitucional cooperativo con el Derecho internacional de los derechos humanos, por interiorizar los derechos universales que les son dados desde fuera, mediante la aplicación e interpretación de las normas constitucionales relativas a los derechos fundamentales de conformidad con los instrumentos internacionales. ${ }^{22}$ Por lo tanto, la apertura constitucional de los Estados mencionados, se da mediante dos vías: una mediante la aplicación de los derechos reconocidos en instrumentos internacionales, y otra, mediante el ejercicio de interpretación de acuerdo al canon internacional de los derechos humanos derivados de tribunales internacionales. Adicionalmente, también se le da valor jurídico a nivel nacional a la Declaración Universal de los Derechos Humanos, pues sin ser propiamente un tratado internacional convencional, al incorporarlo a su ámbito interno le otorgan relevancia jurídica.

De ahí, que lo anterior representó un gran desafió para México, pues el debate nacional se delimitó únicamente sobre el tema de la jerarquía normativa en relación a los tratados internacionales, ${ }^{23}$ no contemplando aspectos como lo es, la interpretación conforme con el uso de la jurisprudencia internacional, el principio pro persona, la aplicación directa de los contenidos de convenciones internacionales. Adicionalmente, es

21 AGUIAR DE LUQUE, Luis. Encuesta sobre los derechos fundamentales. Revista Teoría y Realidad Constitucional, [s.I.], n. 20, p. 14, ener. 2007.

22 HÄBERLE, Peter. El concepto de los derechos fundamentales. In: SAUCA, José Ma. (Ed). Problemas actuales de los derechos fundamentales. Madrid: Universidad Carlos III de Madrid-Boletín Oficial del Estado, 1994. p. 88.

23 Tal es el caso de los diversos criterios establecidos por la Suprema Corte de Justicia de la Nación de México: la jurisprudencia en el que la voz, decía: "LEYES FEDERALES Y TRATADOS INTERNACIONALES. TIENEN LA MISMA JERARQUÍA NORMATIVA [...]" diciembre de 1992. Al igual la jurisprudencia: "TRATADOS INTERNACIONALES. SE UBICAN JERÁRQUICAMENTE POR ENCIMA DE LAS LEYES FEDERALES Y EN UN SEGUNDO PLANO RESPECTO DE LA CONSTITUCIÓN FEDERAL [...]" diciembre de 1992 y la dictada previo a la reforma del año 2011 en materia de derechos humanos, la cual señaló: "TRATADOS INTERNACIONALES. SON PARTE INTEGRANTE DE LA LEY SUPREMA DE LA UNIÓN Y SE UBICAN JERÁRQUICAMENTE POR ENCIMA DE LAS LEYES GENERALES, FEDERALES Y LOCALES. INTERPRETACIÓN DEL ARTÍCULO 133 CONSTITUCIONAL [...]" abril de 2007. MÉXICO. Suprema Corte de Justicia de la Nación. Disponible en http://www2.scjn.gob.mx/red2/2sjt/. Consultado el 2 de junio de 2018. 
oportuno mencionar que nos encontramos actualmente con una realidad innegable por la existencia de una gran interdependencia con otras naciones en diversos campos de la vida económica, social y política de visibles repercusiones e influencia en el ámbito nacional que no pueden dejarse de lado, y con ello, la enorme necesidad de la construcción de un constitucionalismo abierto a las diversas fuentes normativas supranacionales en lo que se refiere a los derechos humanos, pues existía una apertura en otros campos, razón de más para hacerlo en materia de los derechos de las personas. Incluso, cabe mencionar lo que aconteció en el mes de febrero del año 2009, cuando el Consejo de Derechos Humanos de las Naciones Unidas, dentro del Informe Periódico Universal, notificó al gobierno mexicano ochenta y tres recomendaciones, mismas que aceptó, entre las cuales se destacó la urgente necesidad de armonizar la constitución y la legislación local a los estándares internacionales de protección de los derechos humanos. $^{24}$

Por lo tanto, era necesario que la tradición constitucional de México hacia la protección de los derechos fundamentales, que surgió desde el siglo XIX con la creación del amparo mexicano, ahora se pudiera ver fortalecida con el auxilio del canon internacional de los derechos humanos, mediante la clarificación y reconocimiento explícito en la Constitución de la categoría de los tratados internacionales de derechos humanos y su interpretación en sintonía con la jurisprudencia de los tribunales internacionales de la materia, cuando se vulneren los derechos las personas a nivel nacional. Circunstancia que llevaría a México a incorporarse a las nuevas construcciones constitucionales formadas bajo los mandatos de un Estado constitucional cooperativo en el campo de los derechos humanos.

Pues, no bastaba el hecho de que México tuviera el artículo 133 de la Constitución previamente mencionado y ratificados cincuenta y tres tratados internacionales de derechos humanos, ${ }^{25}$ con lo cual se pudiera pensar que, de entrada, se encontraba al día en materia de reconocimiento, sino que era imprescindible un compromiso mucho mayor con el Derecho internacional de los derechos humanos, especialmente con una clarificación de las cláusulas de apertura hacia tal ámbito y el de brindar mecanismos a nivel interno que hagan toda una realidad la garantía de los mismos. Por ello, la imperiosa necesidad de la coherencia y armonía entre el constitucionalismo nacional y el ámbito internacional de los derechos humanos.

Las preocupaciones anteriores, se pensó de alguna manera pudieran estar superadas, cuando en el mes de junio del año 2011, aconteció tal vez una de las

\footnotetext{
24 Consejo de Derechos Humanos de las Naciones Unidas. Disponible en http://www.un.org/spanish/News/ focus.asp?focusID=9. Consultado el 27 de marzo de 2018.

25 La totalidad de los tratados internacionales de derechos humanos y su contenido, se puede ver en: Secretaría de Relaciones Exteriores de México. Disponible en http://www.sre.gob.mx/tratados/. Consultado el 30 de marzo de 2018.
} 
transformaciones constitucionales de mayor calado desde la Constitución mexicana de 1917, en la cual se realizaron una serie de modificaciones y adiciones a la misma, especialmente en su capítulo primero del título primero, para denominarlo: "de los Derechos Humanos y sus Garantías"; en cuyo artículo $1^{\circ}$ reconoce a los tratados de derechos humanos como parte de la Constitución. Asimismo, estableció las reglas de interpretación y aplicación que corresponden a los principios de interpretación conforme y pro persona, con lo que se mostró acorde con los avances del derecho internacional en el ámbito de los derechos humanos, lo que significa un avance en el constitucionalismo nacional, al establecer:

En los Estados Unidos Mexicanos todas las personas gozarán de los derechos humanos reconocidos en esta Constitución y en los tratados internacionales de los que el Estado Mexicano sea parte, así como de las garantías para su protección, cuyo ejercicio no podrá restringirse ni suspenderse, salvo en los casos y bajo las condiciones que esta Constitución establece.

Las normas relativas a los derechos humanos se interpretarán de conformidad con esta Constitución y con los tratados internacionales de la materia favoreciendo en todo tiempo a las personas la protección más amplia.

Todas las autoridades, en el ámbito de sus competencias, tienen la obligación de promover, respetar, proteger y garantizar los derechos humanos de conformidad con los principios de universalidad, interdependencia, indivisibilidad y progresividad. En consecuencia, el Estado deberá prevenir, investigar, sancionar y reparar las violaciones a los derechos humanos, en los términos que establezca la ley. [... . . ${ }^{26}$

En el texto anterior, nos encontramos con diversas cláusulas de apertura al derecho internacional de los derechos humanos, con lo cual, en el plano normativo, la Constitución mexicana, fue muy favorable a la incorporación del canon internacional en materia de derechos fundamentales. Es decir, con dicha reforma se clarifican diversas vías de incorporación del derecho internacional de los derechos humanos, con las cuales, se refuerzan las formas por las que el ordenamiento nacional se ve fortalecido en el plano normativo y jurisprudencial al posibilitar, por un lado, el ampliar el catálogo nacional con la incorporación de los derechos humanos del plano internacional y, por

26 MÉXICO. DECRETO por el que se modifica la denominación del Capítulo I del Título Primero y reforma diversos artículos de la Constitución Política de los Estados Unidos Mexicanos. Diario Oficial de la Federación, D.F., 10 de junio de 2011. Disponible en http://www.dof.gob.mx/nota_detalle.php?codigo=5194486\&fecha $=10 / 06 / 2011$. Consultado el 11 de julio de 2018 . 
otro, al ampliar y modificar las facultades interpretativas con la inclusión de criterios hermenéuticos surgidos en fuente supranacional.

Por tal motivo, en el plano normativo, la multicitada reforma se pone al día con el constitucionalismo contemporáneo, en el sentido de ampliar las vías para la incorporación del Derecho internacional de los derechos humanos, superando con ello, la opción que anteriormente se tenía y que aún prevalece en el artículo 133 de la Constitución, que planteaba exclusivamente el tema de la supremacía constitucional y la jerarquía normativa.

\section{AVANCES Y RESISTENCIAS}

Sin bien es cierto, en párrafos anteriores se mencionó sobre los avances en el plano normativo, derivado de la reforma constitucional en materia de derechos humanos, es pertinente mencionar, cómo ha sido el proceso de materialización e instrumentación de la misma por medio de los diversos operadores jurídicos en México. Para tal propósito, en el presente ejercicio académico se delimita al papel que ha jugado la Suprema Corte de Justicia de la Nación, por ser ésta la última palabra a nivel nacional en materia de justicia y ser sus resoluciones reflejo de la tendencia y debate imperante por la jurisdicción del país.

La primera prueba, en cuanto al comportamiento de nuestro máximo tribunal en relación a la nueva construcción constitucional, fue sin lugar a dudas, el asunto conocido como varios 912/2010, ${ }^{27}$ el cual fue resuelto en el mes de julio del año 2011, a los pocos días de la entrada en vigor de la citada reforma, el cual significó, en opinión de Eduardo Ferrer Mac-Gregor, un importante avance y precedente para establecer en México el control difuso de convencionalidad e incluso el aceptar de igual manera el control difuso de constitucionalidad. ${ }^{28}$ Sin embargo, como el mismo autor lo reconoce, no se comparte el hecho, de que en el citado asunto, la Suprema Corte de Justicia de la Nación resolviera por mayoría de sus miembros, entre otras cosas, que la jurisprudencia de la Corte Interamericana de Derechos Humanos, era de carácter vinculante, sólo en el supuesto en el que el Estado mexicano hubiera sido parte en el litigio, sin embargo, lo limitó cuando el Estado no fuera parte, y lo expresó de la siguiente manera:

\footnotetext{
27 El asunto proviene de una sentencia de la Corte Interamericana de Derechos Humanos conocido como caso Radilla Pacheco vs. Estados Unidos Mexicanos dictado en el año 2009, en la cual se ordenó una serie de medidas de reparación al Estado mexicano, en el que diversas autoridades del país, entre ellas los jueces del Poder Judicial de la Federación, se vieron obligadas a establecer un conjunto de criterios en el sentido de determinar la compatibilidad de una ley con la Constitución y los tratados internacionales (caso particular el Código de Justicia Militar, en relación al fuero militar). CORTE INTERAMERICANA DE DERECHOS HUMANOS. Caso Radilla Pacheco vs. Estados Unidos Mexicanos, excepciones preliminares, fondo, reparaciones y costas, sentencia del 30 de agosto de 2010, serie C, n. 209.
}

28 FERRER MAC-GREGOR, Eduardo. Interpretación conforme y control difuso de convencionalidad. El nuevo paradigma para el juez mexicano. México: UNAM, 2011. p. 385-407. 
Por otro lado, el resto de la jurisprudencia de la Corte Interamericana que deriva de las sentencias en donde el Estado mexicano no figura como parte, tendrá el carácter de criterio orientador de todas las decisiones de los jueces mexicanos [... ..$^{29}$

Lo anterior, es contrario a lo resuelto por distintos tribunales nacionales de países en el ámbito interamericano, en el que la jurisprudencia emitida por la Corte Interamericana, no solamente es de carácter vinculante, sino, que le asignan el valor de norma interpretada e, incluso, la integran al bloque de constitucionalidad, como es el caso específico de la vinculación en Argentina, Republica Dominicana y Perú; de norma interpretada en Costa Rica o el de Bolivia, que la integra al bloque de constitucionalidad. ${ }^{30}$ Pero, sobre todo, el actuar de la Suprema Corte de Justicia de la Nación fue de alguna manera contradictorio a lo establecido en la propia reforma constitucional a su artículo $1^{\circ}$, la posibilidad de realizar la interpretación conforme con los tratados internacionales de las normas relativas a los derechos humanos, es decir, mandata el recurrir al auxilio del canon internacional de los derechos humanos por disposición constitucional, como un cláusula de apertura clara a las fuentes jurisdiccionales en sede internacional.

Sucedió entonces, que la apertura constitucional al orden internacional para la protección de los derechos humanos dada con la reforma de 2011, se puso en riesgo de verse limitada por los criterios impuestos a los órganos de impartición de justicia, en el cual, en el caso de México, la Suprema Corte la sujetó, inicialmente al condicionamiento de la aplicación del derecho internacional de los derechos humanos, siempre y cuando el Estado nacional fuera parte de un litigio internacional. Ante ello, cabe resaltar que las obligaciones internacionales que tienen los Estados les han sido atribuidas, no por la vía impositiva de un orden internacional que no les reconoce autonomía, sino por una decisión que el propio Estado ha tomado en el ejercicio de su soberanía, y que ha manifestado mediante la firma y ratificación de instrumentos internacionales para integrarse a un orden supranacional de protección. Así, este primer paso dado por el máximo tribunal mexicano, reflejó un grado de resistencia hacia las fuentes jurisdiccionales que emanan más allá de las fronteras nacionales.

Lo anterior, fue superado en parte en el año de 2013 por otra de las resoluciones más importantes dictadas por la Suprema Corte de Justicia de la Nación en los últimos años, en la cual, en un tiempo relativamente muy corto, modificó el sentido del carácter vinculante de la jurisprudencia de la Corte Interamericana de Derechos Humanos

29 MÉXICO. Suprema Corte de Justicia de la Nación. Engrose de la Sentencia, asuntos varios 921/2010. Disponible en http://www2.scjn.gob.mx/ConsultaTematica/PaginasPub/DetallePub.aspx?AsuntolD=121589. Consultado el 19 de junio de 2018.

30 Tal comportamiento de tribunales nacionales, la Corte Interamericana los cita de forma literal en el caso de referencia. CORTE INTERAMERICANA DE DERECHOS HUMANOS. Caso Cabrera García y Montiel Flores vs. México, excepciones preliminares, fondo, reparaciones y costas, sentencia del 26 de noviembre de 2010, serie C, n. 220. párrs. 226-231. 
respecto a México, cuando el Estado nacional no sea parte. Sin embargo, en tal resolución surgieron otros aspectos de relevancia significativa, que vuelven a poner en duda la apertura favorable por parte de los operadores jurídicos nacionales hacia el derecho internacional de los derechos humanos. El caso concreto fue la contradicción de tesis 293/2011, resuelta en el mes de septiembre del año 2013.

El referido asunto fue, derivado de dos posturas diferentes, entre la sustentada por el primer tribunal colegiado en materia administrativa y de trabajo del décimo primer circuito y el séptimo tribunal colegiado en materia civil del primer circuito, cuya litis consistió en determinar "(i) la posición jerárquica de los tratados internacionales en materia de derechos humanos en relación con la Constitución; y (ii) el carácter de la jurisprudencia en materia de derechos humanos emitida por la Corte Interamericana de Derechos Humanos"31. La resolución recaída a dicha contradicción de tesis refiere indirectamente a esquemas de jerarquización normativa que no son compatibles con el nuevo régimen constitucional mexicano de protección de los derechos humanos y ha generado una colisión de principios constitucionales que puede llegar a incidir en la garantía y exigencia jurisdiccional en el ámbito nacional de los derechos que el esquema internacional reconoce, protege y garantiza más ampliamente. Toda vez, que con dicha reforma, se establecieron vías para la protección reforzada de los derechos por parte de todos los poderes de la unión, así como de todas las autoridades y servidores públicos que integran el aparato estatal, y que se incluyeron en el artículo $1^{\circ}$ constitucional, como obligaciones a cargo de éstos, la aplicación de instrumentos interpretativos como los principios de interpretación conforme y pro persona para garantizar a las personas progresivamente la protección más amplia de sus derechos.

En la citada resolución, como bien se ha señalado, partió de un paso significativo al decretar que la jurisprudencia de la Corte Interamericana es vinculante sin que sea necesario que el Estado mexicano sea parte, pero, omitió darle ese carácter a las opiniones consultivas o a las sentencias de otros organismos internacionales de los que se ha aceptado su competencia, como lo es la Corte Penal Internacional; adicionalmente, es de resaltar uno de los puntos más álgidos de tal resolución, el hecho de establecer que, si bien, los derechos humanos contenidos en la Constitución y en los tratados internacionales de los cuales México es parte, son el parámetro de control constitucional,

31 PLENO DE LA SUPREMA CORTE DE JUSTICIA DE LA NACIÓN, Contradicción de Tesis 293/2011 entre las sustentadas por el Primer Tribunal Colegiado en materias Administrativa y de Trabajo del Décimo Primer Circuito y el Séptimo Tribunal Colegiado en materia Civil del Primer Circuito, Ministro Ponente: Arturo Zaldívar Lelo De Larrea, México, D.F., 3 de septiembre de 2013, p. 18. MÉXICO. Suprema Corte de Justicia de la Nación. Expediente n. 293/2011. Disponible en http://www2.scjn.gob.mx/ConsultaTematica/PaginasPub/DetallePub. aspx?AsuntolD=129659. Consultado el 10 de julio de 2018. 
pero que si en la Constitución se encuentra una restricción a su ejercicio, prevalecerá esta última, invocando aspectos de disposición soberana del constituyente nacional. ${ }^{32}$

Así, al decretarse las restricciones constitucionales nacionales a las normas internacionales de derechos humanos, se está actuando en contravención con la misma disposición constitucional que establece los principios de interpretación conforme y pro persona, con lo cual se dificulta la garantía jurisdiccional de los derechos humanos.

Sin embargo las actitudes de resistencia de los operadores jurisdiccionales nacionales a las amplias implicaciones en el sistema jurídico mexicano de las cláusulas de apertura contenidas en el artículo $1^{\circ}$ constitucional se continúan presentando, ejemplos claros son algunas referencias al material propuesto por la SCJN como herramienta para la formación en derechos humanos y la difusión del contenido y alcances de la reforma en la materia, en las que después de repasar la doctrina y la jurisprudencia que dan contenido y alcances al término bloque de constitucionalidad y dan fe de su aceptación, ampliación y desarrollo en otros países latinoamericanos donde la tendencia es la atribución de una jerarquía constitucional y el carácter de parámetro de control y validez a las normas de contenido materialmente constitucional aunque no se encuentren integradas a la constitución, máxime tratándose de las normas de fuente supranacional cuando están previstos los principios de interpretación conforme y pro persona para dilucidar la discusión en torno a la relación entre la constitución y los tratados internacionales. No obstante lo anterior, el citado material propuesto por la SCJN menciona:

sin minimizar el mérito propio de cada uno de estos conceptos, hasta la fecha la SCJN no ha adoptado ninguno de ellos, por lo menos en el sentido propuesto por la doctrina nacional o comparada. Con base en los argumentos presentados en las sesiones públicas de la SCJN, la mayoría de los ministros ha rechazado, por motivos diversos, el concepto de bloque de constitucionalidad. En cuanto a la interpretación conforme, la SCJN se ha referido a ésta desde una definición más clásica [...] la judicatura mexicana parece estar construyendo una doctrina propia a través de la cual se determine el contenido y alcance del párrafo segundo del artículo $1^{\circ}$ constitucional [... ..$^{33}$

\footnotetext{
32 PLENO DE LA SUPREMA CORTE DE JUSTICIA DE LA NACIÓN, Contradicción de Tesis 293/2011 entre las sustentadas por el Primer Tribunal Colegiado en materias Administrativa y de Trabajo del Décimo Primer Circuito y el Séptimo Tribunal Colegiado en materia Civil del Primer Circuito, Ministro Ponente: Arturo Zaldívar Lelo De Larrea, México, D.F., 3 de septiembre de 2013, p. 18. MÉXICO. Suprema Corte de Justicia de la Nación. Expediente n. 293/2011. Disponible en http://www2.scjn.gob.mx/ConsultaTematica/PaginasPub/DetallePub. aspx?AsuntolD=129659. Consultado el 10 de julio de 2018.

33 MEDELLÍN URQUIAGA, Ximena. "Principio pro persona” en Metodología para la enseñanza de la reforma constitucional en materia de derechos humanos. México: Suprema Corte de Justicia de la Nación. Tomo l, p. 52. Disponible en http://www.reformadh.org.mx/reformadh/index.php. Consultado el 22 de junio de 2018.
} 
Lo anterior, reafirma la presencia de ciertas resistencias por parte de los operadores jurídicos en México, especialmente el comportamiento de la Suprema Corte de Justicia de la Nación, para asumir a plenitud las disposiciones establecidas en la multicitada reforma constitucional del año 2011, mismas que tienen que ver con la clarificación de las cláusulas de apertura al Derecho internacional de los derechos humanos, pues si bien es cierto, como se ha expresado en el presente documento han realizado pasos importantes para asimilarla y difundirla, no ha sido del todo acorde a la nueva construcción constitucional.

\section{CONCLUSIONES}

Así, a manera de conclusión se señala, que es innegable que vivimos en una realidad global, en la que como bien lo expresa Boaventura de Sousa Santos "los sistemas interestatales modernos fueron diseñados como un sistema de ecúmene jurídica nacional coexistente con una diáspora jurídica internacional", ${ }^{34}$ es decir, es visible que nos encontramos ante nuevas formas de transnacionalización jurídica, en las que los Estados dejaron de ser el centro del monopolio de la producción normativa, sino que, ahora existe una gran diversidad de fuentes que surgen más allá de los ámbitos territoriales de los Estados nacionales, pero que, paradójicamente son las propias constituciones las que habilitan que dicho orden normativo internacional se vuelva parte del ámbito interno. De ahí que una forma de asumir en serio el contenido de los instrumentos internacionales de derechos humanos y lo dispuesto por las cláusulas de apertura hacia ellos, es que los entes nacionales hagan uso de los mismos para resolver dilemas jurídicos internos.

En consecuencia, no existen justificaciones de peso, que legitimen válidamente las resistencias para hacer uso del canon internacional de los derechos humanos, cuando desde el ámbito constitucional nacional se han dispuesto soberanamente cláusulas de conexión hacia este nuevo orden, tal como quedó evidenciado anteriormente. Ante ello, el Estado mexicano normativamente se ha puesto al día, pues no solo habilitó constitucionalmente la incorporación de los contenidos internacionales en materia de derechos humanos, al permitir que una vez ratificados los tratados internacionales de derechos humanos, sean parte del ordenamiento jurídico nacional, incluso en un rango prevalente de nivel constitucional para aplicarse de forma directa, sino que se han dados pasos claros para el uso de un ius commune, especialmente con la posibilidad de que los operadores jurídicos utilicen la figura de la interpretación conforme, para que al momento de la aplicación de un derecho humano, este sea reforzado en su aplicación con el uso de la jurisprudencia internacional de la materia.

34 DE SOUSA SANTOS, Boaventura. Sociología jurídica crítica. Madrid:Trotta, 2009. p. 448. 
Sin embargo, el gran reto que tiene los operadores jurídicos y en general los intérpretes dentro del Estado constitucional, en el que se incluye lo internacional, es cómo se asumen con seriedad los derechos de las personas o qué vías son las necesarias para su cumplimiento, ante el escenario en que nos encontramos, sobre todo con la presencia de resistencias como las expresadas por entes jurídicos mexicanos, que van encaminadas a una cierta idea de volcarse a un constitucionalismos de índole cerrado o con expresiones de corte chauvinistas. Ante ello, la referida premisa de que un Estado se vuelva hacia sus fronteras, sería a todas luces insostenible, pues es ilusorio pensar que por si solo un ente nacional resuelva sus problemas imperantes, por más fuerte que pueda ser, basta mencionar alguno de los dilemas presentes, para evidenciar que un comportamiento cerrado de un país no puede afrontarlos cabalmente, como puede ser el tema migratorio, el terrorismo o el narcotráfico, por mencionar algunos, pues la realidad nos ilustra que no es así, que un Estado no puede ser constitucional, atrincherándose en si mismo. ${ }^{35}$ Así, surge la imperiosa necesidad, de crear espacios en los que los derechos se garanticen, el que se asuman comportamientos favorables a coordinarse entre instancias de diverso signo: universales, regionales o locales, para poner en marcha una serie de acciones de control y límites a los poderes, para remediar los problemas presentes, máxime si se trata de derechos humanos, en el que se conciban estos como una nueva res pública universal y a la vez en parte fundamental del ius commune del constitucionalismo contemporáneo.

\section{REFERENCIAS}

AGUIAR DE LUQUE, Luis. Encuesta sobre los derechos fundamentales. Revista Teoría y Realidad Constitucional, [s.I.], n. 20, p. 11-57, ener. 2007.

ALONSO GARCÍA, Ricardo. Derecho comunitario, derechos nacionales y derecho común europeo. Madrid: Civitas, 1989.

BECERRA RAMÍREZ, Manuel. Recepción del derecho internacional en el derecho interno. México: Universidad Nacional Autónoma de México, 2006.

Consejo de Derechos Humanos de las Naciones Unidas. Disponible en http://www.un.org/spanish/News/focus.asp?focusID=9. Consultado el 27 de marzo de 2018.

CORONA NAKAMURA, Luis Antonio; GÚZMAN ROBLEDO, María Teresa. Evolución y desarrollo de los derechos económicos, sociales y culturales. In: MARTíNEZ LAZCANO, Alfonso Jaime. Derechos económicos, sociales y culturales. Bogotá: Ediciones Nueva Jurídica, 2017.

35 PISARELLO, Gerardo, Globalización, constitucionalismo y derechos. In: CARBONELL, Miguel. VÁZQUEZ, Rodolfo. Estado constitucional y globalización. México: Porrúa, 2003. p. 251-253. 
CORTE INTERAMERICANA DE DERECHOS HUMANOS. Caso Cabrera García y Montiel Flores vs. México, excepciones preliminares, fondo, reparaciones y costas, sentencia del 26 de noviembre de 2010, serie C, n. 220. párrs. 226-231.

CORTE INTERAMERICANA DE DERECHOS HUMANOS. Caso Radilla Pacheco vs. Estados Unidos Mexicanos, excepciones preliminares, fondo, reparaciones y costas, sentencia del 30 de agosto de 2010, serie C, n. 209.

DE ASIS, Rafael. Cuestiones de derecho. Bogotá: Universidad del Externado, 2005.

DE SOUSA SANTOS, Boaventura. Sociología jurídica crítica. Madrid:Trotta, 2009.

FERRER MAC-GREGOR, Eduardo. Interpretación conforme y control difuso de convencionalidad. El nuevo paradigma para el juez mexicano. México: UNAM, 2011.

FIORAVANTI, Mauricio. Constitucionalismo, experiencia histórica y tendencias actuales. Madrid: Trotta, 2014.

GARCÍA RAMÍREZ, Sergio. La "navegación americana" de los derechos humanos: hacia un ius commune. In: VON BOGDANDY, Armin; FIX-FIERRO, Héctor; MORALES ANTONIAZZI, Mariela. Ius constitucionale commune en América Latina: rasgos, potencialidades y desafíos. México: UNAM, Max-Plank-Institut Für Ausländishes e Instituto Iberoamericano de Derecho Constitucional, 2014.

GARCÍA RAMÍREZ, Sergio. La Corte Interamericana de Derechos Humanos. México: Porrúa, 2007.

GARCÍA Y GARCÍA, Antonio. Perspectivas del derecho común romano-canónico al filo del año 2000. In: El derecho común y Europa. Jornadas internacionales de historia del derecho del Escorial de 1999. Madrid: Dykinson, 2000.

HÄBERLE, Peter. El concepto de los derechos fundamentales. In: SAUCA, José Ma. (Ed). Problemas actuales de los derechos fundamentales. Madrid: Universidad Carlos III de Madrid-Boletín Oficial del Estado, 1994.

HABERLE, Peter; KOTZUR, Markus. De la soberanía al derecho constitucional común: palabras clave para un diálogo europeo-latinoamericana. México: UNAM, 2003.

MEDELLÍN URQUIAGA, Ximena. “Principio pro persona” en Metodología para la enseñanza de la reforma constitucional en materia de derechos humanos. México: Suprema Corte de Justicia de la Nación. Tomo I, p. 52. Disponible en http://www.reformadh.org.mx/reformadh/index. php. Consultado el 22 de junio de 2018.

MÉXICO. DECRETO por el que se modifica la denominación del Capítulo I del Título Primero y reforma diversos artículos de la Constitución Política de los Estados Unidos Mexicanos. Diario Oficial de la Federación, D.F., 10 de junio de 2011. Disponible en http://www.dof.gob.mx/nota_detalle.php?codigo $=5194486 \&$ fecha $=10 / 06 / 2011$. Consultado el 11 de julio de 2018 . 
MÉXICO. Suprema Corte de Justicia de la Nación. Engrose de la Sentencia, asuntos varios 921/2010. Disponible en http://www2.scjn.gob.mx/ConsultaTematica/PaginasPub/DetallePub.aspx?AsuntolD=121589. Consultado el 19 de junio de 2018.

MÉXICO. Suprema Corte de Justicia de la Nación. Expediente n. 293/2011. Disponible en http:// www2.scjn.gob.mx/ConsultaTematica/PaginasPub/DetallePub.aspx?AsuntolD=129659. Consultado el 10 de julio de 2018.

MÉXICO. Suprema Corte de Justicia de la Nación. Tesis P. LXXVII/99. Tratados Internacionales. Se ubican jerárquicamente por encima de las leyes federales y en un segundo plano respecto de la Constitución Federal. Registro n. 192867, Semanario Judicial de la Federación y su Gaceta, Tomo X, nov. 1999, p. 46. Tesis Aislada, Novena Época, Pleno, Constitucional.

MÉXICO. Suprema Corte de Justicia de la Nación. Tesis P. IX/2007. Tratados Internacionales. Son parte integrante de la ley suprema de la Unión y se ubican jerárquicamente por encima de las leyes generales, federales y locales. Interpretación del artículo 133 constitucional. Registro n. 172650, Semanario Judicial de la Federación y su Gaceta, Tomo XXV, abr. 2007, p. 6. Tesis Aislada, Novena Época, Pleno, Constitucional.

MÉXICO. Suprema Corte de Justicia de la Nación. Disponible en http://www2.scjn.gob.mx/red2/2sjt/. Consultado el 2 de junio de 2018.

MÉXICO. Tratados Internacionales de Derechos Humanos. Disponible en http://www.un.org/spanish/News/focus.asp?focusID=9. Consultado el 30 de marzo de 2018.

MORÁN MARTíN, Remedios. El ius commune como antecedente jurídico de la Unión Europea. Cuadernos de Historia del Derecho, n. 12, 2005.

PISARELLO, Gerardo, Globalización, constitucionalismo y derechos. In: CARBONELL, Miguel. VÁZQUEZ, Rodolfo. Estado constitucional y globalización. México: Porrúa, 2003.

RUIZ MIGUEL, Alfonso. Entre Estado y cosmópolis. Madrid:Trotta, 2014.

SUÁREZ OSMA, Ingrid. Control de convencionalidad y autoprecedente interamericano. Bogotá: Universidad de la Sabana-Grupo Editorial Ibañez, 2015.

TOMÁS Y VALIENTE, Francisco. Manual de historia de derecho español. Madrid:Tecnos. 2003.

VON BOGDANDY, Armin. lus constitucionale commune latinoamericano. Una aclaración conceptual. In: VON BOGDANDY, Armin; FIX-FIERRO, Héctor; MORALES ANTONIAZZI, Mariela. Ius constitucionale commune en América Latina: rasgos, potencialidades y desafíos. México: UNAM, Max-Plank-Institut Für Ausländishes e Instituto Iberoamericano de Derecho Constitucional, 2014.

ZAGREBELSKY, Gustavo. La ley y su justicia, tres capítulos de justicia constitucional. Madrid: Trotta, 2014.

ZAGREBELSKY, Gustavo. La ley y su justicia. Madrid:Trotta, 2014.

ZAGREBELSKY, Gustavo. La virtud de la duda, una conversación sobre ética y derecho con Geminello Preterossi. Madrid:Trotta, 2012. 